\title{
A New Mixed-Integer Programming Model for Harvest Scheduling Subject to Maximum Area Restrictions
}

\author{
Miguel Constantino \\ Universidade de Lisboa, Faculdade de Ciências, DEIO/Centro de Investigação Operacional, Bloco C6, piso 4, \\ Cidade Universitária,1749-016 Lisboa, Portugal, miguel.constantino@fc.ul.pt \\ Isabel Martins \\ Universidade Técnica de Lisboa, Instituto Superior de Agronomia/Centro de Investigação Operacional, \\ Tapada da Ajuda, 1349-017 Lisboa, Portugal, isabelinha@isa.utl.pt \\ José G. Borges \\ Universidade Técnica de Lisboa, Instituto Superior de Agronomia/Centro de Estudos Florestais, \\ Tapada da Ajuda, 1349-017 Lisboa, Portugal, joseborges@isa.utl.pt
}

\begin{abstract}
Forest ecosystem management often requires spatially explicit planning because the spatial arrangement of harvests has become a critical economic and environmental concern. Recent research on exact methods has addressed both the design and the solution of forest management problems with constraints on the clearcut size, but where simultaneously harvesting two adjacent stands in the same period does not necessarily exceed the maximum opening size. Two main integer programming approaches have been proposed for this area restriction model. However, both encompass an exponential number of variables or constraints. In this work, we present a new integer programming model with a polynomial number of variables and constraints. Branch and bound is used to solve it. The model was tested with both real and hypothetical forests ranging from 45 to 1,363 polygons. Results show that the proposed model's solutions were within or slightly above $1 \%$ of the optimal solution and were obtained in a short computation time.
\end{abstract}

Subject classifications: forest management; harvest scheduling; spatial modeling; integer programming.

Area of review: Environment, Energy, and Natural Resources.

History: Received February 2006; revisions received September 2006, March 2007, May 2007; accepted May 2007.

Published online in Articles in Advance February 20, 2008.

\section{Introduction}

The landscape pattern that results from harvest scheduling has evolved into a critical issue in forest management. Effort in model development has been shifting to better recognize spatial concerns. These concerns have often been addressed within the forest management scheduling adjacency problem. In this problem, stands are considered adjacent if they share a common boundary, and adjacency conflict is defined as a conflict between management alternatives involving a clear-cut in adjacent stands in the same or in consecutive planning periods (Borges and Hoganson 1999). Several authors addressed this combinatorial optimization problem within an integer programming (IP) or mixed integer linear programming (MILP) framework (e.g., Kirby 1980; Covington et al. 1988; Torres Rojo and Brodie 1990; Hof and Joyce 1993; Jones et al. 1991; Yoshimoto and Brodie 1994; Hof et al. 1994; Murray and Church 1996; Snyder and Revelle 1997; Martins et al. 1999, 2005; McDill and Braze 2000, 2001; McDill et al. 2002; Crowe et al. 2003; Murray et al. 2004; Goycoolea et al. 2005). To circumvent computational constraints to the use of exact methods, other authors addressed spatial modeling with heuristics such as
Monte Carlo integer programming (e.g., O'Hara et al. 1989, Nelson et al. 1991), simulated annealing (e.g., Lockwood and Moore 1993, Dahlin and Sallnas 1993), tabu search (e.g., Bettinger et al. 1998, Caro et al. 2003, Richards and Gunn 2003), with dynamic programming-based approaches (e.g., Hoganson and Borges 1998, Borges et al. 1999) or with hybrid heuristic approaches (e.g., Pukkala and Kangas 1993, Boston and Bettinger 2002, Falcão and Borges 2002).

The concept of adjacency may be expanded to address spatial conditions and outcomes of interest other than the clear-cut size. For example, Hoganson et al. (2004) modeled adjacency considerations to meet mature forest interior space goals in Northern Minnesota. Rebain and McDill (2003) modeled minimum mature habitat patch constraints to mitigate fragmentation impacts of strict adjacency. Again both heuristics and exact methods come up as alternative or complementary modeling approaches to target landscape design. However, stand and management unit design itself becomes prominent because it produces the landscape spatial elements upon which decisions are made and thus impacts the spatial conditions targeted by adjacency considerations (Barrett 1997, Borges and Hoganson 1999, Murray 
and Weintraub 2002). In this framework, the area restriction model (ARM) (Murray 1999) emerges as a formulation better suited to address landscape design wider objectives than the traditional unit restriction model formulation. The former includes constraints on opening sizes, and yet it lets the model itself suggest stand aggregation (design) when their combined area does not violate those constraints. Several authors reported heuristic solutions to the ARM (e.g., Lockwood and Moore 1993, Barrett et al. 1998, Clark et al. 2000, Richards and Gunn 2000, Boston and Bettinger 2002, Falcão and Borges 2002). Nevertheless, computational constraints have been an obstacle to effective use of exact methods to address the ARM.

Gunn and Richards (2005) reported good solutions for a relaxed integer programming ARM. Two main integer programming formulations have further been proposed to overcome the computational obstacle. The first approach encompassed an exponential number of constraints (e.g., Martins et al. 1999, McDill et al. 2002, Murray and Weintraub 2002, Crowe et al. 2003). The second approach encompassed an exponential number of variables (e.g., Martins et al. 1999, 2005; McDill et al. 2002; Goycoolea et al. 2005; Vielma et al. 2007). In this paper, we present a new integer programming formulation for the ARM that has a polynomial number of variables and constraints. We start by introducing the notation used to describe the ARM and its main current formulations. An example forest with eight stands is used for illustration purposes. A new model is proposed and tested with both real and hypothetical forests ranging from 45 to 1,363 polygons. Computational efficiency of using the branch and bound to solve the model is reported. Results show that the proposed model's solutions were within or slightly above $1 \%$ of the optimal solution and were obtained in a short computation time.

\section{Area Restriction Model Formulations}

Let a forest be represented by a graph $G=(V, E)$, where each node in $V=\{1, \ldots, N S\}$ corresponds to a stand, and the nodes to each edge in $E$ correspond to two adjacent stands. In this paper, two stands are considered adjacent when both share a boundary that is not a discrete set of points. Thus, the graph $G$ is planar-that is, it can be represented by a figure where the edges do not intersect (Figure 1). See Diestel (2000) for a formal definition and properties of planar graphs. Other definitions of adjacency may be used (Goycoolea et al. 2005, for example, also consider what they call the weak adjacency, where two stands are adjacent if they share a single point at least).

It will be assumed that the forest management planning encompasses the maximization of timber net present value subject to maximum clear-cut size constraints. It will be further assumed that each stand may be harvested only once within the planning horizon, i.e., the minimum rotation is longer than the latter. We will say that a set of stands is contiguous if the corresponding subgraph of $G$ is connected.
EXAMPLE

Figure 1. Graph representation of a forest.
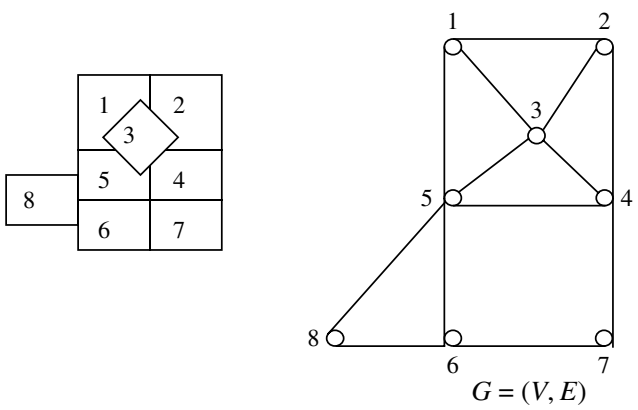

A cluster is a set of contiguous stands. A clear-cut is a cluster such that all stands are harvested in the same period, and no neighboring stand is harvested in the same period.

Let $T=\{1, \ldots, N T\}$ represent the temporal horizon, $a_{i}$ be the area of stand $i$, and $A_{\max }$ be the maximum clear-cut size. $p_{i}^{t}$ corresponds to the timber net present value from stand $i$ if it is harvested in period $t$. These values may be modified to include the value of the end condition of the stands. The ARM for this problem will include constraints stating that the area of each clear-cut cannot exceed $A_{\max }$ (constraints A1). It will further include constraints to ensure that each stand is harvested at most once in the temporal horizon (constraints A2). The ARM model may be further extended to enforce regular timber flows. One may add constraints to preclude periodic fluctuations exceeding a percent value of $v^{t}$, the volume of timber harvested in period $t$, that is, $\left|v^{t+1}-v^{t}\right| \leqslant \Delta v^{t}$ (constraints A3).

Next, we present the two main integer programming formulations discussed in the literature. The first includes stand variables and explicit area restriction constraints. The second includes cluster variables corresponding to feasible clear-cuts. Then, we introduce the new model, which includes stand variables with an additional index to decide on the clear-cut.

\subsection{Formulation with Stand Variables and Explicit Area Restriction Constraints}

In this model (Martins et al. 1999, McDill et al. 2002, Murray and Weintraub 2002, Crowe et al. 2003), stand management options are represented by binary variables:

$x_{i}^{t}= \begin{cases}1, & \text { stand } i \text { is harvested in period } t, \\ 0, & \text { otherwise. }\end{cases}$

To represent constraints $\mathrm{A} 1$, we consider the set $\Re$ of all possible clusters that cannot be harvested as a whole and which are minimal. Each of these clusters is a contiguous group of stands with total size larger than $A_{\max }$ that does not contain any similar cluster. If $r$ is the number of stands in such a cluster, then at most $r-1$ of them can be harvested in the same period. 
EXAmple (Continued). For simplicity, we consider that the area of each stand in our example forest is equal to 1 ha and that the maximum clear-cut area is 2 ha. Thus,

$$
\begin{aligned}
\Re= & \{1-2-3,1-2-4,1-2-5,1-3-4,1-3-5,1-4-5,1-5-6, \\
& 1-5-8,2-3-4,2-3-5,2-4-5,2-4-7,3-4-5,3-4-7, \\
& 3-5-6,3-5-8,4-5-6,4-5-7,4-5-8,4-6-7,5-6-7, \\
& 5-6-8,6-7-8\} .
\end{aligned}
$$

The formulation is as follows (without constraints A3):

$$
\max \sum_{t \in T} \sum_{i \in V} p_{i}^{t} x_{i}^{t}
$$

subject to $\sum_{t \in T} x_{i}^{t} \leqslant 1 \quad \forall i \in V$,

$$
\begin{aligned}
& \sum_{i \in R} x_{i}^{t} \leqslant|R|-1 \quad \forall R \in \Re \text { and } t \in T, \\
& x_{i}^{t} \in\{0,1\} \quad \forall i \in V \text { and } t \in T .
\end{aligned}
$$

Equation (1) expresses the management objective of maximizing the timber harvested net present value. Equation (2) ensures that each stand is harvested at most once (constraints A2). Equation (3) guarantees that in each period at least one stand in each $R \in \Re$ is not harvested (constraints A1). Therefore, each clear-cut will be no larger than the maximum clear-cut area. Equation (4) states the binary requirement on the variables.

The main drawback of this formulation is the huge number of constraints. It may grow exponentially with the number of stands. Nevertheless, if $A_{\max }$ is not too large when compared to the area of most stands (say, three or four times larger), the number of constraints may not be too large, especially if the graph is planar. This is the case for most test instances considered in the literature that further discusses the computational costs of solving more complex problems (e.g., McDill et al. 2002, Murray and Weintraub 2002).

\subsection{Formulation with Cluster Variables}

In this model (Martins et al. 1999, 2005; McDill et al. 2002; Goycoolea et al. 2005; Vielma et al. 2007), decision variables represent feasible clear-cuts. Let $\mathfrak{\Im}$ denote the set of all clusters with area not exceeding $A_{\max }$. Then, the cluster variables will be

$z_{F}^{t}= \begin{cases}1, & \text { cluster } F \in \Im \text { is harvested in period } t, \\ 0, & \text { otherwise. }\end{cases}$

Example (Continued). In the case of the eight-stand example forest, we will have

$$
\begin{aligned}
\Im= & \{1,2,3,4,5,6,7,8,1-2,1-3,1-5,2-3,2-4,3-4,3-5, \\
& 4-5,4-7,5-6,5-8,6-7,6-8\} .
\end{aligned}
$$

The formulation is as follows (without constraints A3):

$$
\begin{aligned}
\max & \sum_{t \in T} \sum_{F \in \mathfrak{I}} p_{F}^{t} z_{F}^{t} \\
\text { subject to } & \sum_{t} \sum_{F: i \in F} z_{F}^{t} \leqslant 1 \quad \forall i \in V, \\
& \sum_{F \cap\{i, j\} \neq \varnothing} z_{F}^{t} \leqslant 1 \quad \forall\{i, j\} \in E \text { and } t \in T, \\
& z_{F}^{t} \in\{0,1\} \quad \forall F \in \mathfrak{I} \text { and } t \in T,
\end{aligned}
$$

where $p_{F}^{t}=\sum_{i \in F} p_{i}^{t}$ is the net present value for cluster $F$ in period $t$. Equation (5) expresses the management objective of maximizing the timber harvested net present value. Equation (6) ensures that each stand is harvested at most once (constraints A2). Equation (7) guarantees that in each period if a cluster is selected to be harvested, then no adjacent clusters or clusters with overlapping stands may be harvested. This prevents the formation of large clusters with area larger than $A_{\max }$ (constraints A1). Equation (8) states the binary requirement on the variables.

This model can be enhanced by substituting Equation (7) with a set of stronger inequalities, the so-called clique constraints (Goycoolea et al. 2005). A clique is a complete subgraph of $G$; it is maximal if it is not contained in any other clique. Let $Q$ be the set of all subsets of nodes that generate maximal cliques.

Example (Continued). In the case of our example forest,

$$
\begin{aligned}
Q=\{ & \{1,2,3\},\{1,3,5\},\{2,3,4\},\{3,4,5\}, \\
& \{4,7\},\{5,6,8\},\{6,7\}\} .
\end{aligned}
$$

Also, Equation (7) can be replaced by

$$
\sum_{F \cap P \neq \varnothing} z_{F}^{t} \leqslant 1 \quad \forall P \in Q \text { and } t \in T .
$$

These constraints are stronger than (7) in the sense that the set of points $\mathbf{z}$ satisfying $\left(7^{\prime}\right)$ is contained in the set of points defined by (7), but not vice versa, yielding, in general, better linear programming relaxation bounds, and more efficient solving by branch and bound.

The main drawback of this model is the large number of variables. It may grow exponentially with the number of stands. Nevertheless, if $N C$ is the maximum number of stands of any feasible cluster, the number of variables is bounded by a polynomial of degree $N C$. This allows this model to have a reasonable performance for some test instances. Goycoolea et al. (2005) reported solutions within $1 \%$ of the optimum for some test forests. The average number of stands per cluster in these instances was 3.02 to 5.05. Vielma et al. (2005) were able to improve these results through sophisticated computational enhancements.

Next, we introduce a new integer programming formulation for the ARM, with a number of variables and constraints bounded by a polynomial in the number of stands NS. 


\subsection{The New Formulation (Area Restriction with Stand-Clear-Cut Variables-ARMSC)}

In this model, there is no definition of any kind of regions a priori. Observe that the number of clear-cuts in the forest in each time period is less than the number of stands $(N S)$. Thus, we can represent the class of clear-cuts in the forest as $\mathcal{C}=\left\{C_{1}, \ldots, C_{N S}\right\}$, where some sets $C_{i}$ can be empty. The particular choice of the stands in $C_{1}, \ldots, C_{N S}$ is left to the model instead of being given a priori as in the cluster formulation. We will consider decision variables:

$y_{j}^{i t}= \begin{cases}1 & \text { if stand } j \text { belongs to set } C_{i} \text { in period } t \\ 0 & \text { otherwise. }\end{cases}$

To express constraints A1, we introduce another set of variables. For each period $t \in T, C_{i} \in \mathcal{C}$ and $e \in E$, let

$w_{e}^{i t}= \begin{cases}1 & \begin{array}{l}\text { if at least one of the stands in edge } e \\ \text { belongs to set } C_{i} \text { in period } t,\end{array} \\ 0 & \text { otherwise. }\end{cases}$

Note that for $e=\left\{j_{1}, j_{2}\right\}, j_{1}$ and $j_{2}$ cannot belong to distinct clear-cuts in the same period $t$, so at most one of the variables $w_{e}^{i t}$ has the value one for each $e$ and $t$.

We consider the following model (without constraints A3):

$$
\max \sum_{t \in T} \sum_{j \in V} p_{j}^{t} \sum_{i \in V} y_{j}^{i t}
$$

subject to $y_{j}^{i t}-w_{e}^{i t} \leqslant 0$

$$
\begin{aligned}
& \forall e \in E, \quad j \in e, i \in V, \text { and } t \in T, \\
& \sum_{i \in V} w_{e}^{i t} \leqslant 1 \quad \forall e \in E \text { and } t \in T, \\
& \sum_{t \in T} \sum_{j \in V} a_{j} y_{j}^{i t} \leqslant A_{\max } \quad \forall i \in V, \\
& \sum_{t \in T} \sum_{i \in V} y_{j}^{i t} \leqslant 1 \quad \forall j \in V, \\
& y_{j}^{i t} \in\{0,1\} \quad \forall j \in V, i \in V, \text { and } t \in T, \\
& w_{e}^{i t} \geqslant 0 \quad \forall e \in E, i \in V, \text { and } t \in T .
\end{aligned}
$$

Equation (9) expresses the management objective of maximizing the timber harvested net present value. Equation (10) defines the relationship between variables $y$ and $w$. Equation (11) ensures that in each period every two adjacent stands are in one clear-cut at most. Equation (12) guarantees that each clear-cut does not exceed the maximum size requirement. Equations (11) and (12) guarantee that constraints A1 are satisfied. Equation (13) states that each stand is harvested at most once in the temporal horizon (constraints A2). Actually, constraints (12) could be replaced by the weaker $\sum_{j \in V} a_{j} y_{j}^{i t} \leqslant A_{\max } \forall i \in V, t \in T$, which state that in each time period no clear-cut is larger than $A_{\max }$. In the presence of constraints (13), each clearcut can occur at most once in the time horizon, so we can add up the left-hand sides of these constraints for $t \in T$ and obtain (12). The remaining equations state binary and nonnegativity requirements on the variables. Observe that the integrality of variables $y_{j}^{i t}$, together with Equation (10), implies the integrality of variables $w_{e}^{i t}$ in at least one optimal solution. Volume constraints (A3) will be included later in this model.

It is important to note that the solutions to this model may not immediately give the set of clear-cuts in each time period. Because there are no constraints enforcing the connectivity of the sets $C_{i}$, they may correspond to disconnected regions with total area not exceeding $A_{\max }$. However, each of these regions is composed by one or more connected regions, that is, clear-cuts with area not exceeding $A_{\max }$. Because the objective function coefficients depend only on the choice of the stands harvested in each time period, any solution with a disconnected harvested set $C_{i}$ is equivalent to the solution where $C_{i}$ is replaced by its connected components. For example, consider example 1, with $A_{\max }=2$. Suppose that we have a feasible solution to the above model with $C_{1}=\{1,7\}, C_{8}=\{8\}$, and $C_{i}=\varnothing$ for $i=2, \ldots, 7$ in some time period. $C_{1}$ corresponds to a disconnected region. Then, the solution with $C_{1}=\{1\}$, $C_{7}=\{7\}, C_{8}=\{8\}$, and $C_{i}=\varnothing$ for $i=2, \ldots, 6$ is equivalent, and each nonempty set $C_{i}$ corresponds to a clear-cut.

To preclude a large number of equivalent solutions (corresponding to all possible orderings of the elements of the set $\mathcal{C}$ ), we may assume that set $C_{i}$ contains stand $i$, and does not contain any stand $j$ with $j<i$. Now each set $C=\left\{i_{1}, \ldots, i_{p}\right\}$ has one and only one representative in $\mathcal{C}$, which is $C_{i}$ with $i=\min \left\{i_{1}, \ldots, i_{p}\right\}$. The following variables may then be eliminated: $y_{j}^{i t}$ if $j<i ; w_{e}^{i t}$ if $e=\left\{j_{1}, j_{2}\right\}$, $j_{1}<i$ and $j_{2}<i$. Equation (10) can also be removed if $j<i$. Moreover, we may introduce the following equations to strengthen the formulation:

$$
\begin{array}{cl}
y_{j}^{i t}-y_{i}^{i t} \leqslant 0 \quad & \forall i, j \in V, j \geqslant i, \text { and } t \in T, \\
w_{e}^{i t}-y_{i}^{i t} \leqslant 0 \quad & \forall i \in V, \quad e=\left\{j_{1}, j_{2}\right\} \in E, \\
& \text { with } i \leqslant j_{1} \text { or } i \leqslant j_{2} \text { and } t \in T, \\
\sum_{j \geqslant i} a_{j} y_{j}^{i t} \leqslant A_{\max } \times y_{i}^{i t} \quad \forall i \in V \text { and } t \in T .
\end{array}
$$

Equations (16) state that if $y_{j}^{i t}=1$ (stand $j$ is in set $C_{i}$ ), then $y_{i}^{i t}=1$ (i.e., $i$, the smallest index stand in $C_{i}$, is also selected to this set). Observe that $y_{i}^{i t}=1$ if and only if set $C_{i}$ is selected to be harvested in period $t$. Equations (17) force variables $w_{e}^{i t}$ to be zero if set $C_{i}$ is not selected in that period. Equations $\left(12^{\prime}\right)$ are justified because $y_{j}^{i t}=0$ whenever $y_{i}^{i t}=0$. To see that $\left(12^{\prime}\right)$ dominates (12), just take the sum of both sides of $\left(12^{\prime}\right)$ for $t \in T$ to obtain the same left-hand side as (12), and a right-hand side not larger than one. Let ARMSC be the formulation defined by (9)-(11), $\left(12^{\prime}\right)$, and (13)-(17). 
This formulation could be easily extended to encompass features such as green-up exclusion period length larger than one period and average area restrictions (e.g., Murray et al. 2004).

If the green-up exclusion time length corresponds to $s$ periods, then constraints (11) should be replaced by $\sum_{i \in V} \sum_{t-s<t^{\prime} \leqslant t} w_{e}^{i t^{\prime}} \leqslant 1 \forall e \in E$ and $s \leqslant t \leqslant N T$.

Average area restrictions state that the total area harvested divided by the total number of clear-cuts should not exceed some value, $A_{\text {ave }}$, in each period. This can be written in the formulation as $\sum_{i, j \in V} a_{j} y_{j}^{i t} \leqslant A_{a v e} \sum_{i \in V} y_{i}^{i t}$ for all periods $t$.

Formulation ARMSC has $\mathrm{O}(N S \times N E \times N T)$ variables and constraints, where $N S$ is the number of stands (nodes of $G$ ), $N E$ is the number of adjacencies (edges of $G$ ), and $N T$ is the number of periods in the planning horizon. If $G$ is planar, the number of edges is of the order of the number of nodes (Diestel 2000), so in this case the formulation has $\mathrm{O}\left(N S^{2} \times N T\right)$ variables and constraints. Even though the number of variables is polynomial, it can be very large for large instances. However, most variables have the value zero in any feasible solution, and this can be determined a priori. Let a chain in $G$, between nodes $i$ and $j$, be a sequence of nodes $\left\{i_{1}, \ldots, i_{p}\right\}$ such that $i_{1}=i, i_{p}=j$, $\left\{i_{k}, i_{k+1}\right\} \in E$ and $i_{k}>i$ with $1<k \leqslant p$. Let the weight of the chain $\left\{i_{1}, \ldots, i_{p}\right\}$ be $\sum_{k=1}^{p} a_{i_{k}}$. If the shortest weight chain between nodes $i$ and $j(i<j)$ has weight larger than $A_{\max }$, then the area of any clear-cut that includes $i$ and $j$ would be larger than $A_{\max }$. In this case, $i$ and $j$ will never be in the same clear-cut and, thus, $y_{j}^{i t}=0$ in any feasible solution, so variables $y_{j}^{i t}$ can be removed from the formulation for all $t \in T$. We can also remove variables $w_{e}^{i t}$ for any edge $e$ such that $y_{j}^{i t}=0 \forall j \in e$, i.e., edges with no nodes in the set $C_{i}$. The elimination of these variables implies the reduction of the number of constraints (10) and (16).

Similarly to the formulation with cluster variables, a tighter formulation for the proposed approach may be obtained replacing edges by cliques. Here the edge variables $w_{e}^{i t}, e \in E$, are replaced by clique variables $W_{P}^{i t}$, $P \in Q$, where $Q$ is the set of maximal cliques of $G$. Let

$$
w_{P}^{i t}=\left\{\begin{array}{l}
1 \quad \begin{array}{l}
\text { if at least one of the stands in clique } P \\
\text { belongs to } C_{i} \text { in period } t
\end{array} \\
0 \quad \text { otherwise. }
\end{array}\right.
$$

Then, Equations (10) and (11) are replaced by the following equations:

$$
\begin{aligned}
& y_{j}^{i t}-W_{P}^{i t} \leqslant 0 \quad \forall P \in Q, j \in P, i \leqslant j, \text { and } t \in T, \\
& \sum_{i \in V} W_{P}^{i t} \leqslant 1 \quad \forall P \in Q \text { and } t \in T .
\end{aligned}
$$

Equations $\left(10^{\prime}\right)$ and $\left(11^{\prime}\right)$ ensure that, in each period, the stands in each maximal clique belong to at most one clearcut. Let $P$ be the set of nodes of a maximal clique with more than two stands, and let $e$ denote an edge in $P$. By Equation $\left(10^{\prime}\right)$, we have $W_{P}^{i t} \geqslant \max _{j \in P} y_{j}^{i t} \geqslant \max _{j \in e} y_{j}^{i t}=$ $w_{e}^{i t}$ for at least an optimal solution $\mathbf{y}$. Thus, $\sum_{i \in V} w_{e}^{i t} \leqslant$ $\sum_{i \in V} W_{P}^{i t} \leqslant 1$, so $\left(10^{\prime}\right)-\left(11^{\prime}\right)$ are stronger than $(10)-(11)$. Variables $W_{P}^{i t}$ can be removed for any $P$ such that variables $y_{j}^{i t}$ are removed $\forall j \in P$. The elimination of these variables implies the reduction of the number of Equations $\left(10^{\prime}\right)$ and $\left(11^{\prime}\right)$.

Finally, the timber flow constraints A3 can be written as

$$
\begin{array}{r}
(1-\Delta) \sum_{j \in V} V_{j}^{t-1}\left(\sum_{i \leqslant j} y_{j}^{i, t-1}\right) \\
\leqslant \sum_{j \in V} V_{j}^{t}\left(\sum_{i \leqslant j} y_{j}^{i t}\right) \leqslant(1+\Delta) \sum_{j \in V} V_{j}^{t-1}\left(\sum_{i \leqslant j} y_{j}^{i, t-1}\right) \\
\forall t=2, \ldots, N T .
\end{array}
$$

These constraints impose, for each period, that the volume of timber harvested is within $\Delta \%$ of that harvested in the previous period.

We will refer to the formulation ARMSC with volume constraints as ARMSCV. When we have clique variables and Equations $\left(10^{\prime}\right)$ and $\left(11^{\prime}\right)$, we refer to those formulations as ARMSC-C and ARMSCV-C, respectively.

\section{Results}

The main objective of the computational tests is to assess the ability of a commercial MIP solver to obtain solutions of a certain quality (say, less than $1 \%$ deviation from the optimum) in a reasonable amount of time (up to two hours) with this model. Solutions within these limits have been obtained for all the instances considered.

As a secondary objective, we want to determine the impact on the quality of the solutions obtained due to some variations on the model.

We report results for both real and hypothetical test forests. Real test forests include Leiria National Forest (LNF) in Portugal and the El Dorado forest in the United States. We give a short description of LNF; El Dorado is referred to in Goycoolea et al. (2005). LNF is a public forest located in the Portuguese central region by the Atlantic Ocean. It extends over approximately 11,000 ha, of which 8,700 have been allocated to a timber production division. The remaining area is managed for conservation purposes (e.g., wind protection) and was not considered in this study. Maritime pine (Pinus pinaster Ait.) even aged high forest stands occupy most of the area allocated for timber production. This forest division was classified into 574 stands according to ecological and productive criteria. Stand area ranges from 1 to 33 ha. The current distribution of stand area by age class is very irregular, with over $50 \%$ of the area with an age between 10 and 34 years. The site index ranges from 12 to $24 \mathrm{~m}$ (base age of 50 years). A typical prescription at LNF encompasses natural regeneration and/or plantation, leaving about 2,000 seedlings 
per ha and a noncommercial thinning at 15 years of age that leaves about 1,500 trees per ha. In the period from 15 to 50 years of age, pine stands are thinned every five years. We have considered a cutting age ranging from 50 to 100 years. The forest supplies two timber products. Logs with larger diameters resulting from clear-cuts are a very valuable asset in LNF. However, there is also demand for material with smaller diameters, resulting mostly from thinnings and from younger rotations. For each stand, timber yields were predicted following the models in Falcão (1997).

In the case of the hypothetical test forests, the graph of the forest map has one of the configurations in Figure 2. For these instances, the area of each stand is equal to 1 ha. The stand age at the beginning of a planning horizon extending to seven 10-year periods was generated randomly between 0 and 100. For each stand, timber yields were predicted following the models in Falcão (1997). Thinnings were not considered in the hypothetical forests. The minimum cutting age is 40 years.

We also report results for two other instances available at the website www.unbf.ca/fmos/, Bloedel and WLC (El Dorado is in this site as well). For Bloedel, the area of each stand is equal to 1 ha. For WLC, the minimum cutting age is also 40 years.

The parameter $\Delta$ used in the timber flow constraints A3 is 0.15 . In the case of Leiria, Bloedel, WLC, and hypothetical test forests, the coefficients of the objective function include the values for the ending conditions of stands.

An important parameter with impact on the size of the formulation is the ratio $A_{\max } / \bar{a}$, where $\bar{a}$ is the average area of the stands. Cluster and cell models grow exponentially with this value. The growth is polynomial for the model presented in this paper. We have considered values of that ratio ranging in the interval $[3,6]$.

The forests had contrasting sizes (Table 1) to better test the proposed approach. The number of stands ranged from 45 to 1,363 and the number of edges from 98 to 3,609 .

The number of variables and constraints in ARMSC and its variants grows polynomially with the number of stands, but it can be extremely large for large instances (see Table 2). Thus, we applied the variable reduction based on stand numbering and shortest chains, as described earlier.

Figure 2. Graph configuration of the type- $F$ instances and the graph of instance G10x2.

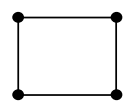

Instances $\mathrm{F}$

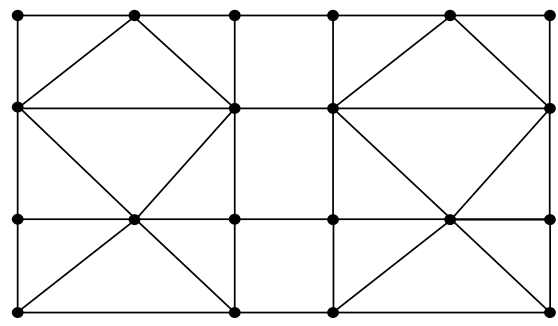

G10x2
Note. The graph of this instance is used to exemplify the graph configuration of the type-G instances.
Table 1. Size of the instances ( $\bar{a}$ is the average of the stand's area).

\begin{tabular}{lrrrccc}
\hline Instance & $\begin{array}{c}\text { No. } \\
\text { nodes }\end{array}$ & $\begin{array}{c}\text { No. } \\
\text { edges }\end{array}$ & $\begin{array}{c}\text { No. } \\
\text { cliques }\end{array}$ & $A_{\max }$ (ha) & $\bar{a}$ (ha) & $A_{\max } / \bar{a}$ \\
\hline Leiria & 574 & 1,152 & 740 & 52 & 14.96 & 3.46 \\
El Dorado & 1,363 & 3,609 & 2,041 & 40 & 12.78 & 3.13 \\
F10x10 & 100 & 180 & 180 & $3 ; 4$ & 1 & $3 ; 4$ \\
F15x15 & 225 & 420 & 420 & $3 ; 4$ & 1 & $3 ; 4$ \\
F20x20 & 400 & 760 & 760 & $3 ; 4$ & 1 & $3 ; 4$ \\
F25x25 & 625 & 1,200 & 1,200 & $3 ; 4 ; 5 ; 6$ & 1 & $3 ; 4 ; 5 ; 6$ \\
G15x7 & 105 & 247 & 145 & $3 ; 4$ & 1 & $3 ; 4$ \\
G40x10 & 400 & 988 & 596 & $3 ; 4$ & 1 & $3 ; 4$ \\
G40x14 & 560 & 1,396 & 844 & $3 ; 4$ & 1 & $3 ; 4$ \\
G60x10 & 600 & 1,492 & 904 & $3 ; 4 ; 5 ; 6$ & 1 & $3 ; 4 ; 5 ; 6$ \\
Bloedel & 45 & 112 & 37 & 4 & 1 & 4 \\
WLC & 73 & 98 & 63 & 40 & 10.12 & 3.95 \\
\hline
\end{tabular}

The proposed procedures for the variable/constraint reduction had a substantial impact on problem sizes (Table 2). In most cases, the reduction ((no. before - no. after)/no before $\times 100)$ was larger than $90 \%$ for the number of both variables and constraints.

We present the results of tests for the following models: ARMSC in Table 3 (no volume flow constraints, no clique variables); ARMSC-C in Table 4 (similar to the previous one but with clique variables $W$ instead of edge variables $w$ ); ARMSCV-C in Table 5 (with volume flow constraints and clique variables); and ARMSCV-C with constraints (12) replacing constraints $\left(12^{\prime}\right)$ in Table 6.

The test problems were solved with CPLEX 9.0 (ILOG 2003) as both linear and integer programming solver. The branch-and-bound algorithm was allowed to run for two hours at most. CPLEX default parameters were used throughout. Computations were done on a desktop computer with an Intel Pentium M-1.6 GHz processor and with 512 MB RAM.

The quality of the integer solutions for each problem instance was measured using the deviation (in percentage) of its value (vis) from the best upper bound found (bup) by the branch-and-bound algorithm: gap $=($ bup $-v i s) /$ vis $\times 100$. The values of bup and gap are given by CPLEX. To further assess the strength of a formulation, we compared the objective function values of the integer solution (vis) and of its linear relaxation (vlrs): gapL $=(v l r s-v i s) /$ vis $\times 100$. Observe that these gaps are upper bounds on the true gaps, $($ opt $-v i s) / o p t \times 100$ and $(v l r s-o p t) / o p t \times 100$, respectively, where opt is the optimal value of the instance.

The algorithm was able to solve the ARMSC for all problem instances except for El Dorado, G40x14_7_4, and G60x10_7_4, where a solution within $1 \%$ of the optimum was obtained at an early node (Table 3 ). The formulation ARMSC-C was effective in two of these three cases, which is not surprising because the corresponding linear relaxation is tighter than the linear relaxation of the ARMSC (Tables 3 and 4). Naturally, the linear relaxations of the ARMSC and the ARMSC-C produced the same results for instances $\mathrm{F}$ because the maximal cliques used in 
Table 2. Size of the ARMSCV-C before and after the variable/constraint reduction.

\begin{tabular}{|c|c|c|c|c|c|c|c|c|}
\hline \multirow[b]{2}{*}{ Instance } & \multirow[b]{2}{*}{$T$} & \multirow[b]{2}{*}{$A_{\max }(\mathrm{ha})$} & \multicolumn{2}{|c|}{ No. variables } & \multirow[b]{2}{*}{ Reduction (\%) } & \multicolumn{2}{|c|}{ No. constraints } & \multirow[b]{2}{*}{ Reduction (\%) } \\
\hline & & & Before & After & & Before & After & \\
\hline Leiria_6_52 & 6 & 52 & $4,525,416$ & 87,210 & 98.1 & $10,691,756$ & 192,386 & 98.2 \\
\hline El Dorado_12_40 & 12 & 40 & $55,675,824$ & 457,332 & 99.2 & $154,508,297$ & $1,056,149$ & 99.3 \\
\hline F10x10_7_3 & 7 & 3 & 196,000 & 15,701 & 92.0 & 450,072 & 32,641 & 92.7 \\
\hline F10x10_7_4 & 7 & 4 & 196,000 & 25,361 & 87.1 & 450,072 & 53,473 & 88.1 \\
\hline F15x15_7_3 & 7 & 3 & $1,015,875$ & 38,171 & 96.2 & $2,343,627$ & 79,316 & 96.6 \\
\hline F15x15_7_4 & 7 & 4 & $1,015,875$ & 63,511 & 93.7 & $2,343,627$ & 133,818 & 94.3 \\
\hline F20x20_7_3 & 7 & 3 & $3,248,000$ & 70,441 & 97.8 & $7,512,532$ & 146,341 & 98.1 \\
\hline F20x20_7_4 & 7 & 4 & $3,248,000$ & 118,811 & 96.3 & $7,512,532$ & 250,263 & 96.7 \\
\hline F25x25_7_3 & 7 & 3 & $7,984,375$ & 112,511 & 98.6 & $18,497,787$ & 233,716 & 98.7 \\
\hline F $25 \times 25 \_7 \_4$ & 7 & 4 & $7,984,375$ & 191,261 & 97.6 & $18,497,787$ & 402,808 & 97.8 \\
\hline F25x25_7_5 & 7 & 5 & $7,984,375$ & 288,057 & 96.4 & $18,497,787$ & 614,894 & 96.7 \\
\hline F25x25_7_6 & 7 & 6 & $7,984,375$ & 400,995 & 95.0 & $18,497,787$ & 865,522 & 95.3 \\
\hline G15x7_7_3 & 7 & 3 & 183,750 & 18,298 & 90.0 & 458,302 & 40,458 & 91.2 \\
\hline G15x7_7_4 & 7 & 4 & 183,750 & 30,002 & 83.7 & 458,302 & 68,297 & 85.1 \\
\hline G40x10_7_3 & 7 & 3 & $2,788,800$ & 80,577 & 97.1 & $7,007,384$ & 177,428 & 97.5 \\
\hline G40x10_7_4 & 7 & 4 & $2,788,800$ & 140,714 & 95.0 & $7,007,384$ & 319,332 & 95.4 \\
\hline G40x14_7_3 & 7 & 3 & $5,503,680$ & 115,129 & 97.9 & $13,855,840$ & 253,608 & 98.2 \\
\hline G40x14_7_4 & 7 & 4 & $5,503,680$ & 202,370 & 96.3 & $13,855,840$ & 459,492 & 96.7 \\
\hline G60x10_7_3 & 7 & 3 & $6,316,800$ & 123,445 & 98.0 & $15,887,140$ & 271,596 & 98.3 \\
\hline G60x10_7_4 & 7 & 4 & $6,316,800$ & 217,490 & 96.6 & $15,887,140$ & 493,244 & 96.9 \\
\hline G60x10_7_5 & 7 & 5 & $6,316,800$ & 336,084 & 94.7 & $15,887,140$ & 774,714 & 95.1 \\
\hline G60x10_7_6 & 7 & 6 & $6,316,800$ & 473,438 & 92.5 & $15,887,140$ & $1,109,503$ & 93.0 \\
\hline Bloedel_3_4 & 3 & 4 & 11,070 & 2,712 & 75.5 & 28,645 & 6,562 & 77.1 \\
\hline WLC_7_40 & 7 & 40 & 69,496 & 4,606 & 93.4 & 145,650 & 9,304 & 93.6 \\
\hline
\end{tabular}

ARMSC-C are the edges used in ARMSC. In some of the other instances, the linear relaxations of the ARMSC-C are tighter than the linear relaxations of the ARMSC.

In the case of the ARMSCV-C, the algorithm was able to find a solution within $1 \%$ of the optimum for all instances tested before (Table 5). The optimum linear relaxation solution is very close to the best integer solution obtained for all instances except for Bloedel. Branch and bound hardly improved the upper bounds provided by the linear relaxations. For some problem instances where the value of $A_{\max }$ was increased, the algorithm was able to find a solution within or slightly above $1 \%$ of the optimum.

Replacing constraints $\left(12^{\prime}\right)$ by constraints (12) in the ARMSCV-C formulation slightly decreased, on average, the quality of the best integer solution (Table 6). The algorithm was able to find a solution within $1 \%$ of the optimum

Table 3. Computational results to the ARMSC and its linear relaxation.

\begin{tabular}{llrcrc}
\hline Instance & $\begin{array}{c}\text { Final } \\
\text { gap }(\%)\end{array}$ & $\begin{array}{c}\text { Sol. time } \\
(\mathrm{sec} .)\end{array}$ & $\begin{array}{c}\text { 1st sol.with gap } \leqslant \\
1 \% / \text { time }(\mathrm{sec} .)\end{array}$ & $\begin{array}{c}\text { Best sol. } \\
\text { time (sec.) }\end{array}$ & GapL $(\%)$ \\
\hline Leiria_6_52 & 0 & 149 & $0.86 / 8$ & 138 & 0.37 \\
El Dorado_12_40 & 0.02 & 7,200 & $0.23 / 405$ & 4,711 & 0.06 \\
F10x10_7_3 & 0 & $<1$ & $0.54 /<1$ & $<1$ & 0.49 \\
F10x10_7_4 & 0 & $<1$ & $0.64 /<1$ & $<1$ & 0.08 \\
F15x15_7_3 & 0 & 1 & $0.07 / 1$ & 1 & 0.04 \\
F15x15_7_4 & 0 & 16 & $0.91 / 2$ & 16 & 0.23 \\
F20x20_7_3 & 0 & 2 & $0 / 2$ & 2 & 0.64 \\
F20x20_7_4 & 0 & 26 & $0.07 / 14$ & 24 & 0.3 \\
F25x25_7_3 & 0 & 28 & $0.98 / 4$ & 20 & 0.55 \\
F25x25_7_4 & 0 & 191 & $0.43 / 46$ & 132 & 0.4 \\
G15x7_7_3 & 0 & 2 & $0.27 / 1$ & 2 & 0.53 \\
G15x7_7_4 & 0 & 7 & $0.9 / 4$ & 104 & 0.77 \\
G40x10_7_3 & 0 & 152 & $0.29 / 31$ & 2,825 & 0.46 \\
G40x10_7_4 & 0 & 5,829 & $0.64 / 132$ & 131 & 0.46 \\
G40x14_7_3 & 0 & 198 & $0.43 / 27$ & 2,389 & 0.6 \\
G40x14_7_4 & 0.05 & 7,200 & $0.43 / 130$ & 107 & 0.56 \\
G60x10_7_3 & 0 & 131 & $0.24 / 40$ & 1,559 & 0.85 \\
G60x10_7_4 & 0.29 & 7,200 & $0.66 / 171$ & 18 & 5.47 \\
Bloedel_3_4 & 0 & 18 & $0.7 / 17$ & $<1$ & 0 \\
WLC_7_40 & 0 & $<1$ & $40 /<14$ & \\
\hline
\end{tabular}


Table 4. Computational results to the ARMSC-C and its linear relaxation.

\begin{tabular}{|c|c|c|c|c|c|}
\hline Instance & $\begin{array}{c}\text { Final } \\
\text { gap }(\%)\end{array}$ & $\begin{array}{l}\text { Sol. time } \\
\text { (sec.) }\end{array}$ & $\begin{array}{c}1 \text { st sol. with gap } \leqslant \\
1 \% / \text { time (sec.) }\end{array}$ & $\begin{array}{l}\text { Best sol. } \\
\text { time (sec.) }\end{array}$ & GapL (\%) \\
\hline Leiria_6_52 & 0 & 174 & $0.73 / 21$ & 165 & 0.34 \\
\hline El Dorado_12_40 & 0 & 4,989 & $0.15 / 328$ & 4,980 & 0.03 \\
\hline F10x10_7_3 & 0 & $<1$ & $0.54 /<1$ & $<1$ & 0.49 \\
\hline F10x10_7_4 & 0 & 1 & $0.64 / 1$ & 1 & 0.08 \\
\hline F15x15_7_3 & 0 & 1 & $0.07 / 1$ & 1 & 0.04 \\
\hline F15x15_7_4 & 0 & 9 & $0.81 / 3$ & 9 & 0.23 \\
\hline F20x20_7_3 & 0 & 2 & $0.82 / 2$ & 1 & 0.64 \\
\hline F20x20_7_4 & 0 & 17 & $0.92 / 4$ & 17 & 0.3 \\
\hline F $25 \times 25$-7_3 & 0 & 28 & $0.16 / 18$ & 26 & 0.55 \\
\hline F $25 \times 25 \_7 \_4$ & 0 & 261 & $0.43 / 52$ & 223 & 0.4 \\
\hline G15x7_7_3 & 0 & $<1$ & $0 /<1$ & $<1$ & 0.49 \\
\hline G15x7_7_4 & 0 & 7 & $0.96 / 5$ & 7 & 0.77 \\
\hline G40x10_7 73 & 0 & 58 & $0.12 / 27$ & 48 & 0.33 \\
\hline G40x10_7_4 & 0.03 & 7,200 & $0.6 / 111$ & 2,418 & 0.55 \\
\hline G40x14_7_3 & 0 & 208 & $0.3 / 28$ & 141 & 0.38 \\
\hline G40x14_7_4 & 0.03 & 7,200 & $0.53 / 139$ & 4,728 & 0.59 \\
\hline G60x10_7_3 & 0 & 48 & $0.82 / 8$ & 46 & 0.53 \\
\hline G60x10_7_4 & 0.27 & 7,200 & $0.71 / 155$ & 5,167 & 0.83 \\
\hline Bloedel_3_4 & 0 & 14 & $1 / 14$ & 8 & 3.79 \\
\hline WLC_7_40 & 0 & $<1$ & $0 /<1$ & $<1$ & 0 \\
\hline
\end{tabular}

for all instances except for F 25x25. Probably, it does not matter whether one uses constraints $\left(12^{\prime}\right)$ or $(12)$.

The time taken for the preprocessing is very short, a few seconds only in the larger instances.

\section{Conclusions}

The ARM may be used to effectively address landscape design objectives. It includes constraints on opening sizes and yet it lets the model itself suggest stand aggregation (design) when their combined area does not violate those constraints. It thus provides a framework for expanding the concept of adjacency to address forest ecosystem management spatial objectives. However, computational constraints have been an obstacle to effective use of exact methods rather than heuristics to solve complex ARM problems. Current models to address the forest management

Table 5. Computational results to the ARMSCV-C and its linear relaxation.

\begin{tabular}{|c|c|c|c|c|c|}
\hline Instance & $\begin{array}{c}\text { Final } \\
\text { gap }(\%)(1)\end{array}$ & $\begin{array}{l}\text { Sol. time } \\
\text { (sec.) }\end{array}$ & $\begin{array}{c}1 \text { st sol. with gap } \leqslant \\
1 \% / \text { time (sec.) }\end{array}$ & $\begin{array}{l}\text { Best sol. } \\
\text { time (sec.) }\end{array}$ & GapL $(\%)$ \\
\hline Leiria_6_52 & 0 & 1,004 & $0.08 / 47$ & 846 & 0.15 \\
\hline El Dorado_12_40 & 0.15 & 7,200 & $0.15 / 2,371$ & 2,371 & 0.16 \\
\hline F10x10_7_3 & 0.2 & 7,200 & $0.78 / 1,798$ & 5,646 & 0.24 \\
\hline F10x10_7_4 & 0.1 & 7,200 & $0.11 / 554$ & 1,218 & 0.22 \\
\hline F15x15_7_3 & 0.78 & 7,200 & $0.97 / 325$ & 4,331 & 0.89 \\
\hline F15x15_7_4 & 0.78 & 7,200 & $0.79 / 3,053$ & 7,086 & 0.79 \\
\hline F20x20_7_3 & 0.29 & 7,200 & $0.86 / 817$ & 6,922 & 0.48 \\
\hline F20x20_7_4 & 0.24 & 7,200 & $0.97 / 24$ & 6,804 & 0.24 \\
\hline F $25 \times 25 \_7 \_3$ & 0.44 & 7,200 & $0.88 / 546$ & 4,414 & 0.65 \\
\hline F25x25_7_4 & 0.36 & 7,200 & $0.57 / 1,072$ & 3,935 & 0.36 \\
\hline F25x25_7_5 & 1.22 & 7,200 & - & 4,105 & 1.22 \\
\hline F25x25_7_6 & 1.1 & 7,200 & - & 6,219 & 1.1 \\
\hline G15x7_7_3 & 0.52 & 7,200 & $0.71 / 3,750$ & 6,903 & 0.71 \\
\hline G15x7_7_4 & 0.7 & 7,200 & $0.83 / 3,250$ & 5,889 & 0.73 \\
\hline G40x10_7 73 & 0.85 & 7,200 & $0.85 / 2,515$ & 2,515 & 0.88 \\
\hline G40x10_7_4 & 0.88 & 7,200 & $0.88 / 4,401$ & 4,401 & 0.88 \\
\hline G40x14_7_3 & 0.24 & 7,200 & $0.74 / 2,875$ & 6,659 & 0.25 \\
\hline G40x14_7_4 & 0.6 & 7,200 & $0.64 / 1,617$ & 3,321 & 0.6 \\
\hline G60x10_7_3 & 0.26 & 7,200 & $0.7 / 30$ & 6,917 & 0.27 \\
\hline G60x10_7_4 & 1 & 7,200 & $1 / 398$ & 398 & 1 \\
\hline G60x10_7_5 & 0.5 & 7,200 & $0.5 / 7,136$ & 7,136 & 0.5 \\
\hline G60x10_7_6 & 1.13 & 7,200 & - & 333 & 1.13 \\
\hline Bloedel_3_4 & 0 & 178 & $1 / 132$ & 161 & 3.32 \\
\hline WLC_7_40 & 0.04 & 7,200 & $0.0 /<1$ & 2,186 & 0.1 \\
\hline
\end{tabular}


Table 6. Computational results to the ARMSCV-C with constraints (12) replacing constraints $\left(12^{\prime}\right)$ and its linear relaxation.

\begin{tabular}{|c|c|c|c|c|c|c|}
\hline Instance & $\begin{array}{c}\text { Final gap }(\%) \\
\text { (2) }\end{array}$ & $\begin{array}{l}\text { Sol. time } \\
\quad \text { (sec.) }\end{array}$ & $\begin{array}{c}1 \text { st sol. with gap } \leqslant \\
1 \% / \text { time (sec.) }\end{array}$ & $\begin{array}{l}\text { Best sol. } \\
\text { time (sec.) }\end{array}$ & GapL $(\%)$ & $(2)-(1)$ \\
\hline Leiria_6_52 & 0 & 4,035 & $0.13 / 37$ & 1,079 & 0.20 & 0 \\
\hline El Dorado_12_40 & 0.2 & 7,200 & $0.2 / 1,676$ & 1,676 & 0.22 & 0.05 \\
\hline F10x10_7_3 & 0.32 & 7,200 & $0.7 / 4,806$ & 5,948 & 0.39 & 0.12 \\
\hline F10x10_7_4 & 1.18 & 7,200 & - & 6,093 & 1.31 & 1.17 \\
\hline F15x15_7_3 & 0.95 & 7,200 & $0.97 / 1,895$ & 1,898 & 1.06 & 0.17 \\
\hline F15x15_7_4 & 0.76 & 7,200 & $0.89 / 1,328$ & 6,851 & 0.77 & -0.02 \\
\hline F20x20_7_3 & 0.57 & 7,200 & $0.92 / 601$ & 4,819 & 0.86 & 0.28 \\
\hline F20x20_7_4 & 0.67 & 7,200 & $0.93 / 19$ & 5,127 & 0.67 & 0.43 \\
\hline F25x25_7_3 & 0.26 & 7,200 & $0.94 / 410$ & 6,691 & 0.56 & -0.18 \\
\hline F25x25_7_4 & 1.02 & 7,200 & - & 6,668 & 1.02 & 0.66 \\
\hline F25x25_7_5 & 1.42 & 7,200 & - & 98 & 1.42 & 0.2 \\
\hline F25x25_7_6 & 2.12 & 7,200 & - & 147 & 2.12 & 1.02 \\
\hline G15x7_7_3 & 0.5 & 7,200 & $0.85 / 91$ & 824 & 0.77 & -0.02 \\
\hline G15x7_7_4 & 0.63 & 7,200 & $0.63 / 3,364$ & 3,364 & 0.66 & -0.07 \\
\hline G40x10_7_3 & 0.35 & 7,200 & $0.91 / 794$ & 5,910 & 0.39 & -0.5 \\
\hline G40x10_7_4 & 0.92 & 7,200 & $0.92 / 4,960$ & 4,960 & 0.93 & 0.04 \\
\hline G40x14_7_3 & 0.18 & 7,200 & $0.89 / 866$ & 6,613 & 0.19 & -0.06 \\
\hline G40x14_7_4 & 0.83 & 7,200 & $0.83 / 3,395$ & 3,395 & 0.83 & 0.23 \\
\hline G60x10_7_3 & 0.22 & 7,200 & $0.33 / 983$ & 6,661 & 0.22 & -0.04 \\
\hline G60x10_7_4 & 0.83 & 7,200 & $0.83 / 95$ & 95 & 0.84 & -0.17 \\
\hline G60x10_7_5 & 0.65 & 7,200 & $1 / 105$ & 6,336 & 0.65 & 0.15 \\
\hline G60x10_7_6 & 0.89 & 7,200 & $0.89 / 430$ & 430 & 0.89 & -0.24 \\
\hline Bloedel_3_4 & 0 & 160 & $0.52 / 113$ & 124 & 7.07 & 0 \\
\hline WLC_7_40 & 0.04 & 7,200 & $0.74 / 7$ & 6,919 & 0.1 & 0 \\
\hline Average & & & & & & 0.13 \\
\hline
\end{tabular}

Note. The last column is the difference between the final gaps of the ARMSCV-C with constraints (12) and the ARMSCV-C with constraints (12').

scheduling adjacency problem encompass either an exponential number of variables or an exponential number of constraints.

This paper presents an approach for model building to increase the efficiency of the solution to harvest scheduling problems with maximum clear-cut size constraints. The proposed new formulation to the ARM-area restriction with stand-clear-cut variables (ARMSC) - has a polynomial number of variables and constraints and may thus contribute to overcome current computational obstacles to effective use of exact methods to support forest ecosystem management. The model was tested with both real and hypothetical forests ranging from 45 to 1,363 standpolygons. The results show that the proposed model found solutions within $1 \%$ of the optimal solution in less than two hours in all cases where the average number of stands per clear-cut ranges in the interval $[3,4]$. These results did not change significantly when this value was increased in some of the problem instances.

Addressing successfully the computational complexity of the ARM may contribute for a better representation of current forest ecosystem management problems. Further research will focus on modeling other spatial conditions and outcomes of interest.

\section{Acknowledgments}

This research was partially supported by Centro de Investigação Operacional (through the project POCTI/ISGL/152). The authors thank Andres Weintraub for providing some real test forest data.

\section{References}

Barrett, T. M. 1997. Voronoi tessellation methods to delineate harvest units for spatial forest planning. Canad. J. Forest Res. 27 903-910.

Barrett, T. M., J. K. Gilles, L. S. Davis. 1998. Economic and fragmentation effects of clearcut restrictions. Forest Sci. 44 569-577.

Bettinger, P., J. Sessions, K. N. Johnson. 1998. Ensuring the compatibility of aquatic habitat and commodity production goals in Eastern Oregon with a tabu search procedure. Forest Sci. 44 96-112.

Borges, J. G., H. M. Hoganson. 1999. Assessing the impact of management unit design and adjacency constraints on forest wide spatial conditions and timber revenues. Canad. J. Forest Res. 29 1764-1774.

Borges, J. G., H. M. Hoganson, D. Rose. 1999. Combining a decomposition strategy with dynamic programming to solve the spatially constrained forest management scheduling problem. Forest Sci. 45 201-212.

Boston, K., P. Bettinger. 2002. Combining tabu search and genetic algorithm heuristic techniques to solve spatial harvest scheduling problems. Forest Sci. 48(1) 35-46.

Caro, F., M. Constantino, I. Martins, A. Weintraub. 2003. A 2-opt tabu search procedure for the multiperiod forest harvesting problem with adjacency, greenup, old growth, and even flow constraints. Forest Sci. 49(5) 738-751. 
Clark, M. M., R. D. Mueller, T. P. McDonald. 2000. A three-stage heuristic for harvest scheduling with access road network development. Forest Sci. 46 204-218.

Covington, W. W., D. B. Wood, D. L. Young, D. P. Dykstra, L. D. Garret. 1988. TEAMS: A decision support system for multiresource management. J. Forestry 86(8) 25-33.

Crowe, K., J. Nelson, M. Boyland. 2003. Solving the area-restricted harvest-scheduling model using the branch and bound algorithm. Canad. J. Forest Res. 33(9) 1804-1814.

Dahlin, B., O. Sallnas. 1993. Harvest scheduling under adjacency constraints - A case study from the Swedish sub-alpine region. Scandinavian J. Forest Res. 8 281-290.

Davis, L., K. Johnson, P. Bettinger, T. Howard. 2001. Forest Management to Sustain Ecological, Economic and Social Values, 4th ed. McGrawHill Publishing Company, New York.

Diestel, R. 2000. Graph Theory, 2nd ed. Graduate Texts in Mathematics. Springer-Verlag, Heidelberg, New York.

Falcão, A. O. 1997. DUNAS_A growth model for the National Forest of Leiria. Proc. IUFRO Workshop Empirical and Process-Based Models for Forest Tree and Stand Growth Simulation, Lisbon, Portugal.

Falcão, A. O., J. G. Borges. 2002. Combining random and systematic search procedures for solving spatially constrained forest management scheduling models. Forest Sci. 48(3) 608-621.

Goycoolea, M., A. T. Murray, F. Barahona, R. Epstein, A. Weintraub. 2005. Harvest scheduling subject to maximum area restrictions: Exploring exact approaches. Oper. Res. 53(3) 490-500.

Gunn, E. A., E. W. Richards. 2005. Solving the adjacency problem with stand-centered constraints. Canad. J. Forest Res. 35 832-842.

Hof, J. G., L. A. Joyce. 1993. A mixed integer linear programming approach for spatially optimizing wildlife and timber in managed forest ecosystems. Forest Sci. 39 816-834.

Hof, J., M. Bevers, L. Joyce, B. Kent. 1994. An integer programming approach for spatially and temporally optimizing wildlife populations. Forest Sci. 40 177-191.

Hoganson, H., J. Bixby, S. Bergmann, J. G. Borges. 2004. Large-scale planning to address interior space production: Three case studies from northern Minnesota. Silva Lusitana 12 35-47.

Hoganson, H. M., J. G. Borges. 1998. Using dynamic programming and overlapping subproblems to address adjacency in large harvest scheduling problems. Forest Sci. 44 526-538.

ILOG. 2003. ILOG CPLEX 9.0-User's Manual. ILOG S.A., Incline Village, NV.

Jones, J. G., B. J. Meneghin, M. W. Kirby. 1991. Formulating adjacency constraints in linear optimization models for scheduling projects in tactical planning. Forest Sci. 37 1283-1297.

Kirby, M. W. 1980. A Guide to the Integrated Resources Planning Model. USDA Forestry Services, Berkeley, CA.

Lockwood, C., T. Moore. 1993. Harvest scheduling with spatial constraints: A simulated annealing approach. Canad. J. Forest Res. 23 468-478.
Martins, I., M. Constantino, J. G. Borges. 1999. Forest management models with spatial structure constraints. Working paper 2/1999, Centro de Investigação Operacional, Faculdade de Ciências, Lisbon, Portugal.

Martins, I., M. Constantino, J. G. Borges. 2005. A column generation approach for solving a non-temporal forest harvest model with spatial structure constraints. Eur. J. Oper. Res. 161(2) 478-498.

McDill, M. E., J. Braze. 2000. Comparing adjacency constraint formulations for randomly generated forest planning problems with four age-class distributions. Forest Sci. 46(3) 423-436.

McDill, M. E., J. Braze. 2001. Using the branch and bound algorithm to solve forest planning problems with adjacency constraints. Forest Sci. 47(3) 403-418.

McDill, M. E., S. A. Rebain, J. Braze. 2002. Harvest scheduling with area-based adjacency constraints. Forest Sci. 48(4) 631-642.

Murray, A. T. 1999. Spatial restrictions in harvest scheduling. Forest Sci. 45(1) 45-52.

Murray, A. T., R. L. Church. 1996. Analyzing cliques for imposing adjacency restrictions in forest models. Forest Sci. 42(2) 166-175.

Murray, A. T., A. Weintraub. 2002. Scale and unit specification influences in harvest scheduling with maximum area restrictions. Forest Sci. 48(4) 779-789.

Murray, A. T., M. Goycoolea, A. Weintraub. 2004. Incorporating average and maximum area restrictions in harvest scheduling models. Canad. J. Forest Res. 34 456-464.

Nelson, J. D., J. D. Brodie, J. Sessions. 1991. Integrating short-term, areabased logging plans with long-term harvest schedules. Forest Sci. 37 $101-122$.

O'Hara, A. J., B. H. Faaland, B. B. Bare. 1989. Spatially constrained timber harvest scheduling. Canad. J. Forest Res. 19 715-724.

Pukkala, T., J. Kangas. 1993. A heuristic optimization method for forest planning and decision making. Scandinavian J. Forest Res. 8 560-570.

Rebain, S., M. E. McDill. 2003. A mixed-integer formulation of the minimum patch size problem. Forest Sci. 49 608-618.

Richards, E. W., E. A. Gunn. 2000. A model and tabu search to optimize stand harvest and road construction schedules. Forest Sci. 49(4) 608-618.

Richards, E. W., E. A. Gunn. 2003. Tabu search design for difficult forest management optimization problems. Canad. J. Forest Res. 33 1126-1133.

Snyder, S., C. ReVelle. 1997. Dynamic selection of harvests with adjacency restrictions: The SHARe model. Forest Sci. 43(2) 213-222.

Torres-Rojo, J. M., J. D. Brodie. 1990. Adjacency constraints in harvest scheduling: An aggregation heuristic. Canad. J. Forest Res. 20 978-986.

Vielma, J. P., A. T. Murray, D. M. Ryan, A. Weintraub. 2007. Improving computational capabilities for addressing volume constraints in forest harvest scheduling problems. Eur. J. Oper. Res. 176(2) 1246-1264.

Yoshimoto, A., J. D. Brodie. 1994. Comparative analysis of algorithms to generate adjacency constraints. Canad. J. Forest Res. 24 1277-1288. 
Copyright 2008, by INFORMS, all rights reserved. Copyright of Operations Research is the property of INFORMS: Institute for Operations Research and its content may not be copied or emailed to multiple sites or posted to a listserv without the copyright holder's express written permission. However, users may print, download, or email articles for individual use. 